\title{
The role of immunotherapy in advanced renal cell carcinoma
}

\author{
Ercília Rita Mondlane ${ }^{1}$, Pedro Abreu-Mendes ${ }^{2,3}$, Diana Martins ${ }^{4,5,6,7,8}$, Rui Cruz ${ }^{1}$, Fernando Mendes 4,5,6,7 \\ ${ }^{1}$ Politécnico de Coimbra, ESTeSC, DFARM, ESTeSC, SM Bispo, Coimbra, Portugal; ${ }^{2}$ Serviço de Urologia, \\ Centro Hospital Universitário de São João, Porto, Portugal; ${ }^{3}$ Faculdade de Medicina Universidade \\ do Porto, Porto, Portugal; ${ }^{4}$ Politécnico de Coimbra, ESTeSC, DCBL, SM Bispo, Coimbra, Portugal; 5 \\ Universidade de Coimbra, Instituto de Investigação Clínica e Biomédica de Coimbra Coimbra, Portugal; \\ ${ }^{6}$ Universidade de Coimbra, Centro de Biomedicina e Biotecnologia Inovadoras (CIBB), Coimbra, \\ Portugal; ${ }^{7}$ Centro Académico Clínico de Coimbra (CACC), Coimbra, Portugal; ${ }^{8}$ Instituto de Investigação \\ e Inovação em Saúde, Universidade do Porto, Porto, Portugal
}

\section{INTRODUCTION}

Cancer has become increasingly common worldwide, being the second leading cause of death and an important barrier to increasing life expectancy in all countries in the XXI century (1). The reasons behind these statistic numbers are complex, but they are associated with aging, population growth and the increased prevalence of risk factors (1).

Kidney cancers are ranked 14th in the World among the ones with the highest incidence (1-3). The renal cell carcinoma (RCC) represents $80-85 \%$ of all kidney cancers, and it is the most common and the third most diagnosed urogenital malignancy (2). It occurs usually in the sixth and seventh decades and most commonly in men (4). The incidence varies globally, with the highest rates in developed countries such as North America and Europe and the lowest rates in Asia and Africa (3).

Due to the high incidence and mortality levels of RCC, it is important to find the most appropriate therapeutic strategies, and also to analyse the influence of risk factors. Age (over 85 years), gender (male), smoking habit, analgesics use, obesity, lack of physical activity, exposure to industrial or environmental agents and comorbidities such as hypertension, urinary stones, diabetes, liver and chronic kidney diseases, are known factors related to the incidence of RCC (5). Currently, most of the RCC cases have been diagnosed through computed tomography or abdominal ultrasonography, in asymptomatic subjects (2).

RCC is divided into multiple subtypes according to its histological characteristics. The most common subtype is clear cell renal cell carcinoma (ccRCC) $(2,6)$, responsible for approximately $80 \%$ of all cases of RCC. The other major subtypes include papillary (12\%), chromophobe (4\%), oncocytoma $(4 \%)$ and collecting duct $(<1 \%)$. Familial RCC is often seen in the context of an inherited syndrome, such as Von Hippel-Lindau (VHL) syndrome and Birt-Hogg-Dubé syndrome $(4,6)$.

RCC's treatment can be conducted following two pathways, namely: local treatment with nephrectomy or other ablative strategies (in small masses and older patients), or through systemic therapy; based on the disease staging. In most cases of localized renal cancer, partial or total nephrectomy can be used to eradicate the disease (2). However, the post-operative recurrence rate can be of $20-40 \%$ in the first 5 years and 5-10\% in late recurrence (4). In cases of recurrence and progression after initial surgical treatment during follow-up or in cases of advanced renal cell carcinoma (aRCC) at presentation, 
the best treatment is systemic. Based on a further classification of aRCC as favourable, intermediate or poor prognosis, based on predetermined scores (3), the best systemic therapy varies.

Immunotherapy represents a relatively recent therapeutic approach in cancer treatment. With several advances in the last decade, this particular form of treatment is already considered extremely important in different cancer types (melanomas, lung, head, neck, urethra and kidney cell cancer) (7). Immunotherapy consists of using and enhancement of the immune system itself, for the detection and elimination of cancer cells, generating a durable response and effective regression, in addition to preventing metastases $(6,8,9)$. Immunotherapeutic strategies include the use of immune system modulators, monoclonal antibodies (MAb), vaccines and, more recently, immune checkpoint inhibitors $(7,9$, 10). This study aims to perform a systematic review in the use of the immune system as a therapeutic strategy to treat aRCC as well as its impact on patient survival and quality of life.

\section{MATERIAL AND METHODS}

The literature used in this review is available on the indexed search engine "Pubmed/Medline". The selected key words were "immunotherapy", "advanced renal cell carcinoma", "immune checkpoints inhibitors", "monoclonal antibodies", according to Medical Subject Headings (Mesh). The inclusion and exclusion criteria were created to guarantee the relevance and validity of the information. Therefore, the inclusion criteria were scientific articles and clinical trials (humans) with a publication date equal to or less than 5 years, availability of free-full text. The authors excluded papers in which the title, abstract, and content were not relevant to this study.

The research strategies used are detailed in Figure-1, and all the sources that provided theoretical support were referenced (Figure-1).

\section{COMMENTS}

\section{Cytokines}

Cytokines were the first immunotherapeutic strategy to be used in clinical practice, with the approval of Interferon- $\alpha$ (IFN- $\alpha$ ) in 1986. Injected cytokines directly stimulate the growth and activity of immune cells and there are 3 types of cytokines used in immunotherapy: IFN, interleukins (IL), and granulocyte-macrophage colony-stimulating factor (GM-CSF) (11).

\section{Interferon- $\alpha$}

IFN's mechanism of action is based on the activation of $\mathrm{T}$ and natural killer (NK) cells and cell cycle inhibition (2). IFN- $\alpha$ is classified as a type I IFN and it comprises a family of more than 20 distinct variants, encoded by a cluster on chromosome 9 . For all IFN- $\alpha$ subtypes action, a connection to a specific membrane receptor complex (IFN-AR) is necessary. This binding leads to the activation of intracellular signalling cascades that increase the expression and activation of signal transducers and transcription activators (STAT1, STAT2 and STAT3) (12). STAT1 is the most implicated in cell death programmed by IFN- $\alpha$. IFN-AR are not only expressed in malignant cells, but also in non-neoplastic cells and it makes the risk of developing higher (12) adverse events (AE). A phase III study concluded that only a small number of patients experienced a complete response with IFN- $\alpha$ monotherapy and the AE related made it difficult to evaluate the long-term use (13). Results of the most recent study about the use of IFN- $\alpha$ in aRCC are presented in Table-1.

\section{Interleukin-2}

IL-2, approved by the Food and Drug Administration (FDA) for metastatic kidney cancer and for metastatic melanoma $(3,11)$ acts by stimulating the proliferation of T cells, cytotoxic T lymphocytes (CTL) specific to tumours, NK cells and possibly intratumor lymphocytes (2). These immunological effects occur through binding IL-2 to its receptors (IL-2R). IL-2R have subunits $\alpha, \beta$ and $\gamma$, and can be dimeric (IL-2R $\beta$ $+\mathrm{IL}-2 \mathrm{R} \gamma$ ) or trimeric (IL- $2 \mathrm{R} \alpha+\mathrm{IL}-2 \mathrm{R} \beta+\mathrm{IL}-2 \mathrm{R} \gamma$ ). The association of IL-2R $\alpha$ (CD25), IL-2R $\beta$ (CD122) and IL$2 \mathrm{R} \gamma$ (CD132) subunits result in the trimeric IL-2R $\alpha \beta \gamma$, which has a high affınity for IL-2. In this association, the main function of CD25 is to increase affinity for IL-2, while CD122 and CD132 (mostly expressed in NK, monocytes, macrophages and CD4+ and CD8+ cells) mediate signal transduction. CD25 is extremely important for the proliferation of immunosuppressive, regulating $\mathrm{T}$ cells. However, in its absence, and 
Figure 1 - PRISMA flow diagram of study selection process.

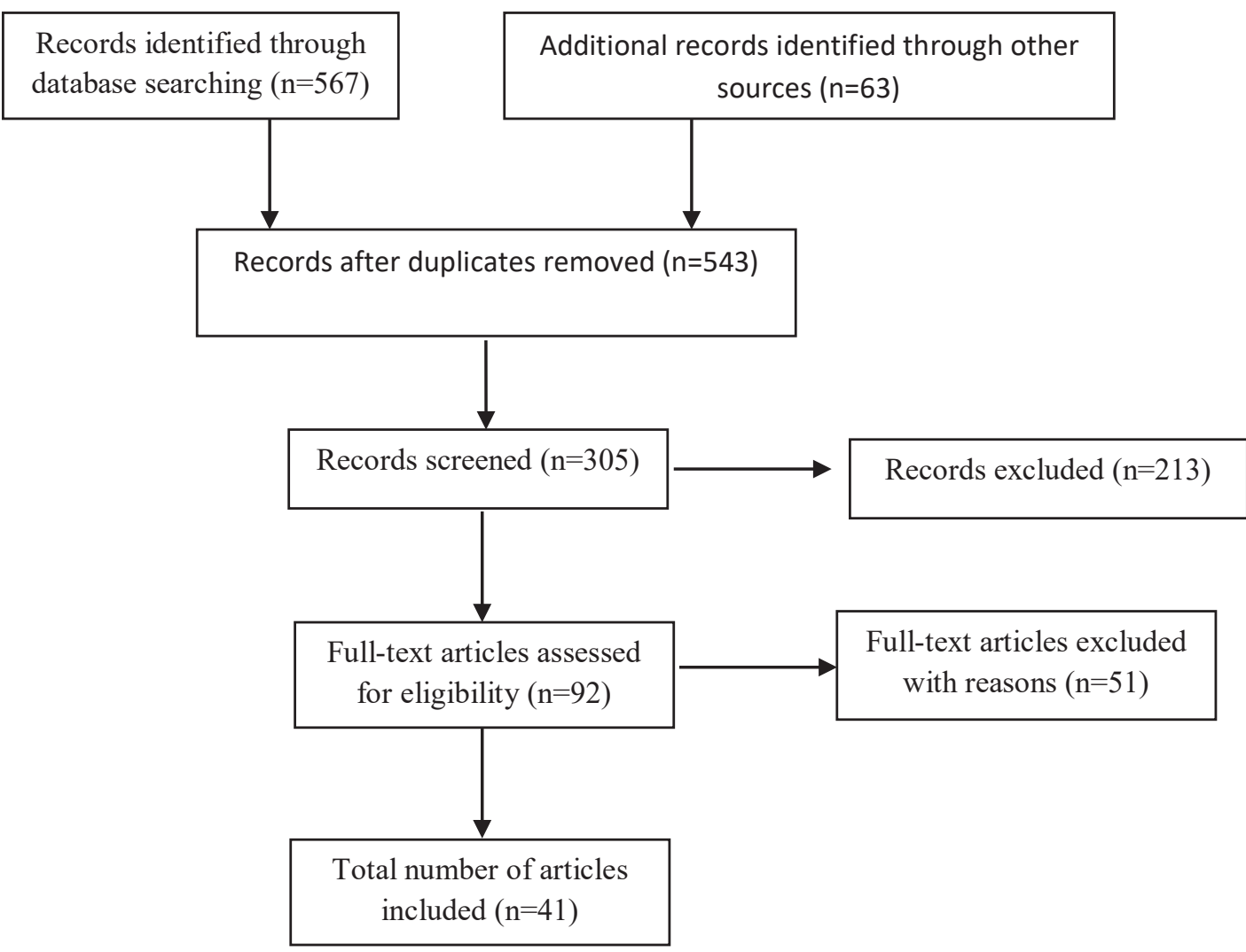

by IL-2R $\beta \gamma$ action, NK and CD8+ cells can be stimulated to proliferate and kill cells that respond to IL-2 $(14,15)$. So, the IL-2 formulations that confer advantage are those that allow binding of IL-2 to CD122 and CD132, but which disfavour the association of IL-2 with CD25 (14, 15). Despite presenting lower toxicity when compared to IFN- $\alpha$, complete and durable results require administration of high doses of IL-2 (HD IL-2) (3). A randomized study performed to compare the outcomes of HD IL-2 and IL-2 showed a greater objective response rate (ORR) (21\% versus $13 \%$ ), response durability and overall survival (OS) in HD IL-2 arm. HD IL-2 was tested in combination with bevacizumab in a phase II study, and the results are shown in Table-1 (13).

\section{Vaccines}

The main objective of the implementation of vaccines in anticancer therapy is the activation of the immune response against cancer cells, overcoming the tolerance generated by the tumour. Ho- wever, not all types of cancer are susceptible to this therapy. Vaccines are implemented in slow-progressing immunogenic cancers that contain specific tissue proteins (16). It is believed that the choice of the target antigen is the most important decision for the development of an anticancer vaccine, because other than non-directed vaccines (such as tumour lysate vaccines), the vast majority of vaccines are designed to generate T-cell responses against shared tumour antigens (those expressed in cancer cells and healthy tissue) (17). There are different types of vaccines: DNA, mRNA, peptide and protein, dendritic cell (DC) and tumour cell vaccines (18). Many significant scientific advances have been made during the last decade, regarding cancer vaccines development (19).

\section{Dendritic Cell Vaccines}

Most of the vaccines under development are essentially intended to promote the presentation of tumour-associated antigens by antigen-presenting cells (APC), to generate long lasting immuni- 
Table 1 - Results of clinical trial articles (conducted between 2015 and 2020) included in the review.

\begin{tabular}{|c|c|c|c|c|}
\hline Drug & Authors/Year & Trial & Results & AE observed (any grade) \\
\hline INF- $\alpha$ & $\begin{array}{l}\text { Eto et al. (2015) } \\
(43)\end{array}$ & $\begin{array}{l}\text { Phase II study evaluated the } \\
\text { combination of IFN- } \alpha \text { with } \\
\text { sorafenib in } 42 \text { patients with } \\
\text { confirmed aRCC. }\end{array}$ & $\begin{array}{l}\text { - ORR was } 26.1 \% \text {; } \\
\text { - Median OS was not reached; } \\
\text { - Grade 3/4 AE were observed } 42 \% \text { of the patients } \\
\text { discontinued treatment due to AE; }\end{array}$ & $\begin{array}{l}\text { Hand foot skin reaction } \\
(64.3 \%) \text {; malaise }(57.1 \%) \text {; rash } \\
(52.4 \%) \text {, diarrhoea }(47.6 \%) \text {; } \\
\text { thrombocytopenia }(45.2 \%)\end{array}$ \\
\hline Interleukin-2 & $\begin{array}{l}\text { Donskov et al. } \\
(2018)(44)\end{array}$ & $\begin{array}{l}\text { Phase II study compared IL-2 plus } \\
\text { IFN- } \alpha \text { plus bevacizumab versus } \\
\text { IL-2 plus IFN- } \alpha \text { in } 118 \text { patients } \\
\text { with favourable or intermediate } \\
\text { risk. }\end{array}$ & $\begin{array}{c}\text { - ORR was } 44.1 \% \text { (IL2+INF+BEV) versus } 28.8 \% \\
\text { (IL2+INF). } \\
\text { - Median OS was } 30.3 \text { months (IL2+INF+BEV) versus } \\
34.1 \text { months (IL2+INF); } \\
\text { - Grade } 3 / 4 \text { AE occurred in } 64 \% \text { (IL2+INF+BEV) } \\
\text { versus } 61 \% \text { (IL2+INF) of the patients }\end{array}$ & $\begin{array}{l}\text { IL+INF+BEV: fatigue (97\%); } \\
\text { flu like symptoms (95\%); } \\
\text { nausea }(90 \%) \text {; dry skin } \\
\text { (71\%); diarrhoea }(64 \%) ; \\
\text { IL2+INF: fatigue }(95 \%) \text {; flu } \\
\text { like symptoms (93\%); nausea } \\
\text { (88\%); dry skin }(81 \%) ; \\
\text { diarrhoea }(73 \%)\end{array}$ \\
\hline AGS-003 & $\begin{array}{l}\text { A. Amin et al. } \\
(2015)(22)\end{array}$ & $\begin{array}{l}\text { Phase II study evaluated the } \\
\text { combination of sunitinib plus } \\
\text { AGS-003 in } 21 \text { patients with } \\
\text { intermediate or poor prognostic. }\end{array}$ & $\begin{array}{l}\text { - No complete responses were observed } 62 \% \\
\text { experienced a clinical benefit ( } 42.9 \% \text { correspond to } \\
\text { partial response and } 19.0 \% \text { to stable disease); } \\
\text { - Median OS was } 30.2 \text { months; } \\
\text { - 42.9\% experienced grade } 3 \mathrm{AE} \text { associated with } \\
\text { sunitinib. No grade } 4 \mathrm{AE} \text { was reported }\end{array}$ & $\begin{array}{l}\text { Diarrhoea }(59 \%) \text {; fatigue } \\
(59 \%) \text {; nausea }(55 \%) \text {; rash } \\
(46 \%) \text {; weight decrease } \\
(41 \%)\end{array}$ \\
\hline IMA901 & $\begin{array}{l}\text { Rini et al. (2016) } \\
\text { (24) }\end{array}$ & $\begin{array}{l}\text { Phase III study (Imprint) } \\
\text { compared the clinical effect of } \\
\text { IMA901 plus sunitinib versus } \\
\text { sunitinib monotherapy in } 139 \\
\text { patients. }\end{array}$ & $\begin{array}{l}\text { - Median OS was } 33.17 \text { months (IMA901+SUN) } \\
\text { versus not reached (SUN); } \\
\text { - } 57 \% \text { of the patients (IMA901+SUN) versus } 47 \% \\
\text { (SUN) experienced grade } 3 / 4 \text { AE. }\end{array}$ & $\begin{array}{c}\text { * IMA901+SUN: } \\
\text { hypothyroidism (27\%); } \\
\text { diarrhoea (26\%); PPE } \\
\text { syndrome (23\%); fatigue } \\
(19 \%) ; \text { nausea (19\%). } \\
\text { SUN: diarrhoea (26\%); } \\
\text { PPE syndrome (25\%); } \\
\text { hypothyroidism (23\%); } \\
\text { fatigue (19\%); hypertension } \\
(18 \%) ;\end{array}$ \\
\hline
\end{tabular}

Atezolizumab Mcdermott et al

(2018) (62)

Rini et al. (2019)

(61)
Phase II (IMmotion150)

compared atezolizumab monotherapy, atezolizumab plus bevacizumab versus sunitinib in 305 patients in ITT and PDL1+populations.
In the ITT population:

- Median ORR was $32 \%$ (ATE+BEV) versus $29 \%$

(SUN) versus $25 \%$ (ATE)

In the PD-L1+population:

- Median ORR was 46\% (ATE+BEV) versus 27\%

(SUN) versus $28 \%$ (ATE);

- Median OS was not presented for both groups;

- Grade $3 / 4$ AE occurred in 40\% (ATE+BEV) versus $57 \%$ (SUN) versus $17 \%$ (ATE).

In the ITT population:

- ORR was $37 \%$ (ATE+BEV) versus $33 \%$ (SUN);

- Median OS was 33.6 months (ATE+BEV) versus

34.9 months (SUN).

In the PD-L1+ population:

- ORR was $43 \%$ (ATE+BEV) versus $35 \%$ (SUN);

- Median OS was 34.0 months (ATE+BEV) versus 32.7 months (SUN);

- Grade 3/4 AE occurred in 40\% (ATE+BEV) versus $54 \%$ (SUN);
Not referred.

ATE+BEV: hypertension

(33\%); fatigue (28\%); diarrhoea $(20 \%)$; proteinuria (20\%); asthenia (15\%).

SUN: diarrhoea (47\%);

PPE syndrome (43\%); hypertension ( $40 \%$ ); fatigue

$(33 \%)$; nausea $(31 \%)$. 
Avelumab (2018) (58)

(2019) (60)

Phase III study (JAVELIN Renal 101) compared the combination of avelumab plus axitinib versus sunitinib as first-line treatment, in 886 patients.

$100)$ evaluated the combination of avelumab plus axitinib as first-line treatment in 55 patients.
Diarrhoea (58\%); dysphonia $(47 \%)$; hypertension (47\%); fatigue (46\%); palmar-plantar erythrodysesthesia syndrome $(31 \%)$;

In the ITT population:

- ORR was 51.4\% (AVE+AXI) versus 25.7 (SUN). In the PD-L+ population: - ORR was $55.2 \%$ (AVE+AXI) versus $25.5 \%$ (SUN); - Grade $3 / 4$ AE occurred in $71.2 \%$ (AVE+AXI)) versus $71.5 \%$ (SUN).

In the first-line treatment: - ORR was $16.1 \%$;

Phase IB study evaluated the use of avelumab monotherapy as first or second line treatment in 82 patients.

AVE+AXI: diarrhoea (62.2\%); hypertension (49.5); fatigue $(41.5 \%)$; nausea (34.1\%); palmar-plantar erythrodysesthesia syndrome $(33.4 \%)$.

SUN: diarrhoea $(47.6 \%)$; fatigue $(40.1 \%)$; nausea (39.2\%); hypertension (36.0\%); PPE syndrome (33.7\%).

In the first-line treatment: pruritus (19.4\%); fatigue (17.7\%); asthenia (14.5\%); nausea $(14.5 \%)$; pyrexia
- Median OS was not reached.

In the second-line treatment: - ORR was $10 \%$;

- Median OS was 16.9 months; - Grade $3 / 4$ AE occurred in $12.9 \%$ (first-line) and $5.0 \%$ (second line). $(12.9 \%)$.

In the second-line treatment: infusion-related $\mathrm{AE}(30.0 \%)$; fatigue $(25.0 \%)$; any immune-related $\mathrm{AE}(15.0 \%)$; diarrhoea $(15.0 \%)$; pyrexia $(10.0 \%)$.

NIV: fatigue (33\%); nausea (14\%); pruritus (14\%);

- ORR was $25 \%$ (NIV) versus $5 \%$ (EVE); (2015) (45) compared nivolumab versus everolimus in 821 previously treated patients.
- Median OS was 25.0 months (NIV) versus 19.6 months (EVE); -Grade $3 / 4$ AE occurred in $19 \%$ of the patients (NIV) and $37 \%$ (EVE). diarrhoea (12\%); decreased appetite (12\%).

EVE: fatigue (34\%); stomatitis $(30 \%)$; diarrhoea (21\%); decreased appetite

$(21 \%)$; rash $(20 \%)$.
Amin et al. (2018)
Phase I study (Checkmate 216) compared the safety and efficacy of nivolumab plus sunitinib versus nivolumab plus pazopanib in 53 patients.
- ORR was 55\% (NIV+SUN) versus 45\% (NIV+PAZ); - Median OS was not reached (NIV+SUN) versus 27.9 months (NIV+PAZ);

- Grade $3 / 4$ AE occurred in $81.8 \%$ (NIV+SUN) versus $70 \%$ (NIV+PAZ).
NIV+SUN: fatigue (84.8\%); diarrhoea (63.6\%); dysgeusia $(63.6 \%)$; nausea $(57.6 \%)$; hypertension $(48.5 \%)$. NIV+PAZ: nausea $(75.0 \%)$; fatigue $(60.0 \%)$; diarrhoea $(60.0 \%)$; dysgeusia $(63.6 \%)$; decreased appetite $(40.0 \%)$ 
Pembrolizumab Atkins et al. (2018)

(54)

Phase IB study evaluated the combination of axitinib plus pembrolizumab in 52 patients.

Rini et al. (2019)

(53)

Phase III (Keynote-426) study compared the combination of pembrolizumab plus axitinib versus sunitinib in 861 treatmentnaïve patients.

Taylor et al. (2020) Phase IB/II evaluated the effect of

(55) Pembrolizumab plus lenvatinib in

30 patients with aRCC after failing previous therapies.

Ipilimumab

Hammers et al.

(2017) (50)
Phase I (CheckMate 016) study evaluated the combination of ipilimumab plus nivolumab in 194 patients. 2 groups of patients were analysed: N3l1 (NIV 3mg/kg plus IPI $1 \mathrm{mg} / \mathrm{kg}$ ) and N1I3 (NIV $1 \mathrm{mg} / \mathrm{kg}$ plus IPI 3mg/kg).
- ORR was $73 \%$;

- Median OS was not reached, but at 18 months, the probability of being alive was $93.9 \%$;

- Grade $3 / 4$ AE occurred in $65 \%$ of the patients.
* Fatigue (63\%); diarrhoea (62\%); dysphonia

(46\%); increased alanine aminotransferase concentration $(29 \%)$; hypertension $(27 \%)$
-ORR was $59.3 \%$ (PEM+AXI) versus $35.7 \%$ (SUN); -At 12 months, the percentage of patients alive was $89.9 \%($ PEM+AXI) versus $78.3 \%$ (SUN); -Grade $3 / 4$ AE occurred in $75.8 \%$ (PEM+AXI) versus $70.6 \%$ (SUN);

- ORR was $70 \%$.

- ORR was $40.4 \% \mathrm{~N} 311$ and $\mathrm{N} 1 \mathrm{l} 3$ groups;

- Median OS was not reached (N3/1) versus 32.6 months (N1/3);

- Grade 3/4 AE occurred in 38\% (N311) versus 61.7\% (N1|3).
PEM+AXI: diarrhoea (54.3\%); hypertension ( $44.5 \%)$; fatigue (38.5\%); hypothyroidism (35.4\%); decreased appetite $(29.6 \%)$

$\phi$ Hypothyroidism (42\%), adrenal insufficiency $(7 \%)$, hypothyroidism (6\%), colitis (4\%), thyroiditis, autoimmune thyroiditis (4\%)

N311: fatigue (51.1\%); rash (31.9\%); pruritus $(31.9 \%)$; nausea $(27.7 \%)$; arthralgia $(25.5 \%)$.

N113: fatigue (68.1\%); nausea (44.7\%); diarrhea (44.7\%); pruritus $(36.2 \%)$; increased lipase $(34.0 \%)$.

-ORR was 39\% (NIV+IPI) versus 31\% (SUN);-Median OS was not reached (NIV+IPI) versus 33.4 months (SUN);

-Grade 3/4 AE occurred in 58\% (NIV+IPI) versus $91 \%$ (SUN).
NIV+IPI: pruritus (26\%): increased lipase $(21 \%)$; pyrexia $(16 \%)$; rash $(16 \%)$, diarrhoea (13\%);

SUN: decreased platelets (85\%); decreased white blood cells (68\%); PPE syndrome $(68 \%)$ decreased appetite (44\%); decreased neutrophils

$(44 \%)$

$\mathbf{A E}=$ adverse events; $\mathbf{a R C C}=$ advanced renal cell carcinoma; $\mathbf{A T E}=$ atezolizumab; $\mathbf{B E V}=$ bevacizumab; $\mathbf{E V E}=$ everolimus; IFN- $\boldsymbol{\alpha}=$ interferon alpha; IL-2 - Interleukin-2; ITT = intention to treat; $\mathbf{N I V}=$ nivulomab; $\mathbf{O R R}=$ objective rate response; $\mathbf{O S}=$ overall survival; $\mathbf{P A Z}=$ pazopanibe; $\mathbf{P D}-\mathbf{L} \mathbf{1}=$ Programmed death-ligand 1; PFS = progression-free survival; PPE = palmar-plantar erythrodysthesia; $\mathbf{S U N}=$ sunitinib

* Only grade 1-2 AE percentages; $\boldsymbol{\phi}$ Total AE percentages for the set of cancers analysed in the study, among which is aRCC. 
Figure 2 - The role of immunotherapy in advanced renal cell carcinoma and its action mechanisms.

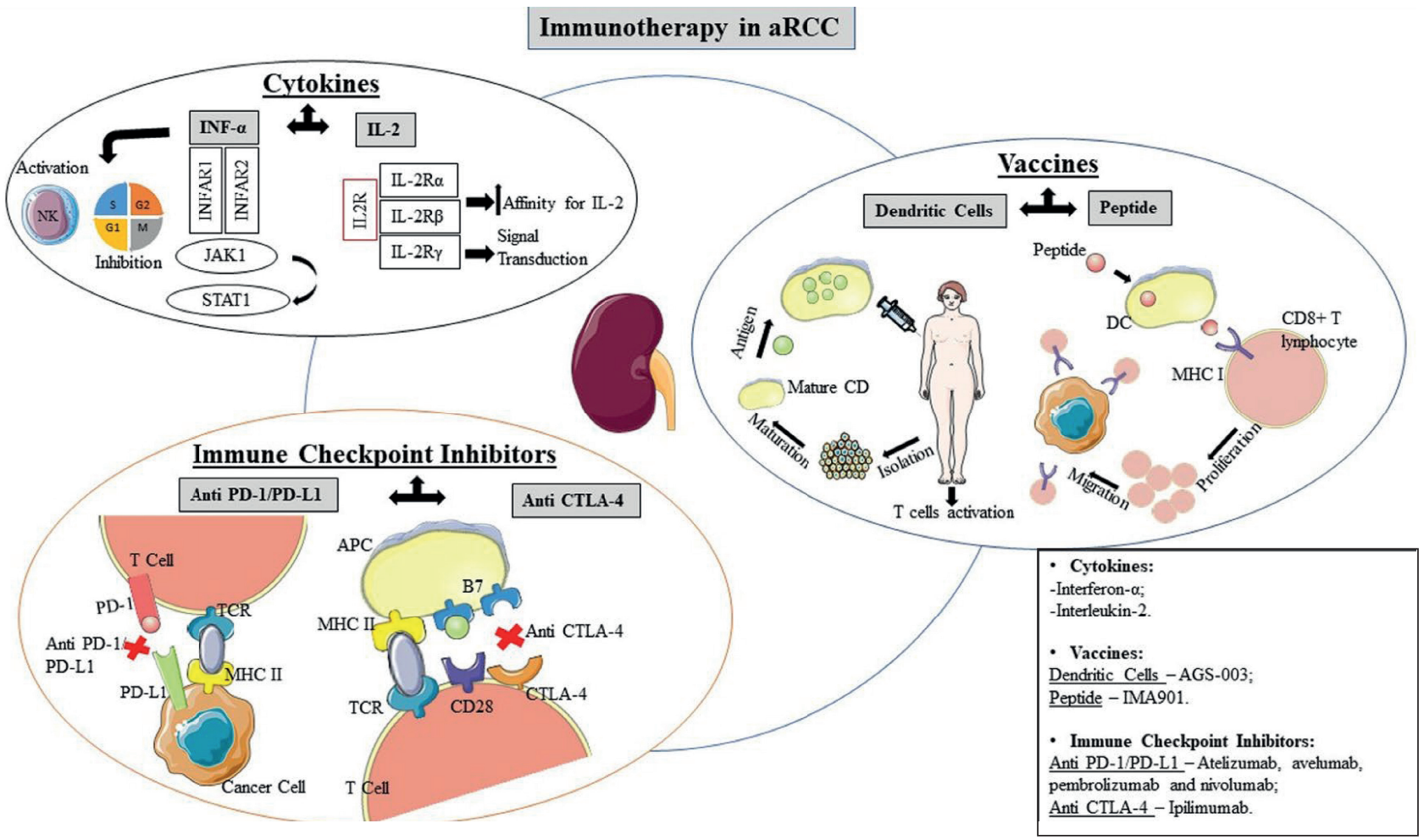

APC - antigen-presenting cells; CD28 - Cluster of differentiation; CTLA-4 - cytotoxic T-lymphocyte-associated protein 4; DC - dendritic cells; IL - interleukin; IFN- $\alpha$ Interferon alpha; IFNAR1 - interferon- $\alpha / \beta$ receptor 1 ; IFNAR2 - interferon- $\alpha / \beta$ receptor 2; JAK1 - Janus kinase 1 ; MHC I - major histocompatibility complex I; MHC II - major histocompatibility complex II; PD-1 - programmed cell death-1; PD-L1 - programmed cell death- Ligand 1; STAT1 - Signal transducer and activator of transcription $1 ;$ TCR - T cell receptor.

ty through t-cell activation. DC are considered the most effective APC, and for this reason, the effective presentation of tumour antigens by these cells is considered an important factor for the development of cancer vaccines (Figure-2) (19). The first cancer vaccine was sipuleucel-T, a DC vaccine, approved in 2010 by the FDA for the treatment of prostate cancer due to its ability to prolong survival $(11,19,20)$.

The immune system can recognize and destroy cells with neoplastic alterations under normal conditions. This mechanism acts as the main defence against cancer cells, and CD8+ T cells are mainly implicated in the process. T cells need tumour antigen presentation made by APC, to stimulate naïve T cell proliferation and differentiation into effector cells. After the recognition of the main antigen complexes class I of the histocompatibility complex (MHC) on the surface of the tumour cell, the T cell tackles cancer cells through different mechanisms, and a subset of specific $\mathrm{T}$ cells for the antigen differen- tiates into memory cells for long-term antitumor protection. DC also contribute to the activation of $\mathrm{T}$ helper cells (CD4+), which are also essential to activate CD8+ T cells (19).

One of the known strategies for DC vaccines development involves the use of the patient's own cells. These cells are first subject to an ex vivo maturation process using toll like receptors (TLR) and agonist cytokines, and then the specific antigens or specific tumour proteins of the patient are loaded. After this process, the cells are injected into the patient in combination with adjuvants, intradermally $(11,19)$.

\section{AGS-003}

AGS-003 is an immunotherapeutic DC vaccine tested for the treatment of aRCC in combination with sunitinib in a phase II (Table-1) and phase III (discontinued) studies. It is made up of autologous dendritic blood cells, generated by tumour-derived 
RNA electroporation and CD40 ligand in host immune cells (13, 21, 22). In aRCC, local and systemic effects generated by the tumour, lead to the production of CD40+ cells. However, DC dysfunction hinders the presentation of antigens and consequently the expected response. The administration of AGS003 helps to circumvent tumour caused effects by presenting mature DC loaded with RNA to produce a more effective and potent response (13).

\section{Peptide Vaccines}

Vaccines based on peptides use protein fragments specifically expressed in tumour cells (18). Peptide vaccines are chemically synthesized, and can be composed by 20-30 amino acids targeting a specific epitope of antigens $(18,23)$. Despite peptides not having negatively charged backbones, such as DNA and mRNA, the use of delivery vehicles is indispensable to maintain stability, ensure direction and minimize undesirable effects (18).

\section{IMA901}

IMA901 vaccine consists of 9 different human lymphocyte antigens (HLA) class I binding-tumour-associated peptides and one HLA class II binding-tumour-associated peptide (24). Because IMA901 has 10 different peptides linked to the tumour, it promotes an expansion of multiple $\mathrm{T}$ cells with different antigen specificities. Induction of CD4+ and CD8+ T-cell responses against tumour-associated antigens causes a broad immune response, although specific against targets functionally relevant to cancer cells. Targeted genes by peptides contained in IMA901 are chosen considering their overexpression in kidney tumour cells, when compared to normal cells (24). IMA901 showed a favourably median OS compared to that obtained in studies with Sunitinib and Sorafenib in a phase II study (25), and a phase III study results (Imprint) are presented in Table-1.

\section{Monoclonal antibodies}

$\mathrm{MAb}$ are laboratory manufactured structures $(9,26)$ to serve as substitute antibodies. They can enhance, restore, or mimic immune system's action. MAb are formed by two heavy and two light polypeptide chains, bonded by a disulphide bond, resulting in the formation of a "y" structure. This structure includes the variable region (FAB), responsible for recognizing specific antigens and the constant region $(\mathrm{FC})$, responsible for binding the antibody to the cells involved in the immune response. Depending on the antigen, the antibody may generate an antibody-dependent cell cytotoxicity or a complement-system cytotoxicity. These responses can generate inhibition of intracellular signals and membrane receptors blockage (27).

Recently, MAb specifically directed to checkpoints between cancer cells and immune system cells, such as cytotoxic T lymphocyte associated protein 4 (CTLA-4), programmed cell death protein and its binding (PD-1/PD-L1) and adoptive T-cell therapy with Chimeric Antigen Receptor T cell receptor (CAR-T) cells, have shown significant clinical benefit in different types of cancer (28).

\section{Checkpoint Inhibitors}

It is known that cancer cells have few antigens "foreign" to the body because they are derived from their own cells. Although cancer cells are immunogenic, the immunological response can be inhibited by factors contained in the tumour (10).

The adaptive immune response initiates recognizing the antigen by the $\mathrm{T}$ cell receptor, with the aid of an APC. This corresponds to the first signal, but to induce cell death, cytokine secretion and memory T-cell formation, a second signal is needed $(10,29,30)$. These events, particularly the amplitude and quality of the response are regulated by the second signal, which is given by the inhibitory and/or excitatory factors known as checkpoints (responsible for inhibiting the exacerbation of the immune response, which may lead to an autoimmune response) $(20,29$, 31). However, in case of a malignant disease, there is a deregulation in these checkpoints expression (29), with an increase in the expression of inhibitory factors that negatively compromise the action of the immune system against cancer.

Immunotherapy based on the regulation of checkpoints has emerged as a promising cancer treatment strategy, showing significant responses to various antigens $(29,32)$ and proving efficiency in the treatment of melanoma, lung cancer, bladder cancer, kidney cell cancer 
and others (33). The most revealing checkpoints studies for cancer treatment include CTLA-4 and PD-1/PD-L1 observed in figure-2 $(3,20,34,35)$.

\section{CTLA-4}

CTLA-4 is the first T cells inhibitory regulator to be identified and tested clinically (30) and it inhibits the response of $\mathrm{T}$ cells in primary phases of its activation. For the activation of these cells, the binding of CD28 with the ligands B7-1 (CD80) and B7-2 (CD26) generates the second signal. CD80 is a dimer with a relative high affınity and CD26 is a monomer with lower affinity for CD28. CTLA-4 can interact with both ligands with higher affinity than CD28. Interaction with these ligands serves to inhibit $\mathrm{T}$ cells response, although the precise mechanisms are not completely understood (36). However, the replacement of CD28 by CTLA- 4 on T cell surface occurs later, thus inactivating its proliferation and function $(6,37)$. Ipilimumab was the furst anti-CTLA-4 to be studied and used in cancer treatment. In aRCC, the combination of ipilimumab plus nivolumab was tested, and results from phase I and III studies are shown in Table-1. Tremelimumab is also a CTLA-4 inhibitor, although less significant since data indicates that its advantages are not superior to that of standard chemotherapy (31).

\section{PD-1/PD-L1}

PD-1 is a molecule expressed on the surface of $\mathrm{T}$ cells that binds to its ligand (PD-L1), found in APC. This interaction between the two molecules regulates the induction and maintains the peripheral pathway $(31,38)$. After initial T cell activation, interactions between PD-1/PD-L1 causes inhibition of its proliferation and cytokines production (Figure-2). Cytoplasmic PD-1 presents a sequence of amino acids involved at the onset of signal transmission; tyrosine is one of these amino acids. When immunoreceptor tyrosine-bases inhibitory motif (ITIM) tyrosine is replaced by phenylalanine, the inhibitory effect generated by PD-1 remains. When immunoreceptor tyrosine-based switch motif (ITSM) tyrosine is replaced by phenylalanine, the inhibitory effect is lost. Therefore, tyrosine in the ITSM region causes the inhibitory effect of PD-1, through recruitment of SHP1 and SHP2. SHP2 in B cells prevents the mobilization of $\mathrm{Ca}$ ions and the phosphorylation of $\mathrm{IgB}$,
SyK, PLC- $\gamma 2$, ERK1 and ERK2. During T cell activity, PD-1 is accumulated near to T cell receptor (TCR), and SHP2 is recruited to the cytosolic domain of PD1 , where it promotes the dephosphorylation of the molecules responsible for TCR signalling (38). The PD-1/PD-L1 pathway also blocks the phosphorylation of ZAP70 and the function of leukocyte-specific tyrosine kinase, leading to inhibition of TCR signalling (38). Atezolizumab, avelumab, pembrolizumab and nivolumab (first monoclonal antibody approved for the treatment of aRCC by the FDA in 2015) are PD-1/PD-L1 inhibitors tested in aRCC (13, 21 ), and the results of its clinical trials are presented in Table- 1 .

\section{DISCUSSION}

The last couple of years have been of utter importance to systemic treatments available for aRCC: the number of approved drugs increased and, most importantly, drugs with better efficacy (39).

Before the use of currently licensed therapies, the treatment of renal cancer was chemotherapy based, with low ORR of approximately 5\% (40). After the chemotherapy failure, investigators started to develop systemic treatment involving the use of immune system (8). Cytokine immunotherapies, such as: IL-2 and IFN- $\alpha$, were established as the standard care, alone or in combination $(4,13)$. The combination of IFN- $\alpha$ plus bevacizumab was approved by the FDA, but it is no longer used as a single agent, due to the advantages of vascular endothelial growth factor (VEGF) targeted therapies as first-line (13). IFN- $\alpha$ and IL-2 can be associated with high level of toxicity (41) but, Curti et al. demonstrated that the development of AE was significantly associated with improved response and tumour control (42).

Although no better results have been provided with the combination of sorafenib and IFN- $\alpha$ (43) and the combination of bevacizumab with IL-2 plus IFN- $\alpha$ (44), this last association combined with sorafenib improved results (55). This proves that potential benefits can arise from the use of cytokines along with other therapies.

Although cancer vaccines improved outcomes, and showed high safety profile (extremely important, because most approved therapies have serious $\mathrm{AE}$ such as cutaneous, gastrointestinal and 
vascular events) (22), they have failed to demonstrate efficacy in phase III studies, despite evidence of immunological activity. Preclinical data show that cancer vaccines have their greatest effect in settings of low or absent tumour volume, suggesting that the success probability as monotherapy would be increased in prophylactic treatment, reducing the incidence of disease $(17,33)$. It is believed that the ex vivo preparation of vaccines may change the functionalities and viability of them, in addition to inefficient delivery, because it is possible that administrated vaccines may not be able to reach their targets with precision $(11,18,19)$. The other suggested reason may be related to the antigen choice and the immunosuppressive nature of the tumour's microenvironment, because neoantigens specific $T$ cells are not subject to an optimal microenvironment. Thus, it is possible that combination of vaccines with other therapies (especially those aimed to the microenvironment), may be an option for improving their effectiveness (17). Amin et al. demonstrated that when the AGS003 was added to sunitinib (first-line treatment for favourable risk), in patients with aRCC with low and intermediate risk, the expected survival was doubled and this combination also presented a good safety profile (22). Curiously, Rini et al. concluded that the combination of sunitinib with IMA901 did not improve relevant outcomes when compared to sunitinib monotherapy (24). The difference between the results might be related to vaccines mechanism of action, since AGS-003 consists of a reinforcement of APC, which helps to stimulate T cells, and IMA901 consists of small fragments of peptides expressed in tumour cells. The contribution of IMA901 becomes ineffective when there is no reinforcement of the APC to help present these antigens. Therefore, the advantages of IMA901 might be clearly expressed in the prevention of recurrences.

In recent years, studies have been developed with more specific immunological agents, which have revolutionized the oncology principles in RCC. The FDA has approved six antibodies that target the PD-1/PD-L1 pathway: atezolizumab, durvalumab and avelumab targeting PD-L1, and cemiplimab, nivolumab and pembrolizumab targeting PD-1 (8).

The Checkmate 025 study showed a significant improvement in the average OS and demonstrated a favourable safety profile (45), which led to an approval of nivolumab in 2015 by the FDA, and in 2016 by the European Medicines Agency (EMA), for patients with aRCC treated with anti-angiogenic agents (46). Stukalin et al. conducted a study that explored the real-world efficacy of nivolumab compared to cabozantinib in the second line setting, concluding that the efficacy was similar for both therapies. This leads to a scenario in which the choice of the therapy to be used as second-line depends more on pragmatic factors, such as: safety profile, availability, price and patient choice (which can be conditioned by the drug's administration that is intravenous, for nivolumab, and oral, for cabozantinib) (47).

A retrospective study conducted by Kimura et al. concluded that there are possibly no differences in the priority of nivolumab or axitinib as second-line treatment, however, they suggest that, comparing to axitinib, nivolumab should be the choice in aRCC patients with comorbidities (48).

The Checkmate016 and Checkmate214 studies showed that the combination of nivolumab and ipilimumab has a manageable safety profile, durable response and higher efficacy when compared with nivolumab monotherapy and sunitinib, respectively (49-51). This combination therapy is recommended to aRCC patients with clear cell pathology and International Metastatic RCC Database Consortium (IMCD) poor/intermediate risk; patients with clear and non-clear cell pathology with sarcomatoid component (52).

Studies conducted with the combination of pembrolizumab plus axitinib and pembrolizumab plus lenvatinib showed improved outcomes and a manageable safety profile (53-55). The combination of pembrolizumab plus axitinib was shown to induce longer OS tolerable in treatment-naïve patients, compared to first-line sunitinib $(52,56)$. The results recommend this combination as the present first-line therapy to patients with clear cell pathology with IMCD favourable, poor/intermediate risk and patients with clear and non-clear cell pathology with sarcomatoid component (52).

The Javelin Renal 101 study demonstrated that the combination of avelumab plus axitinib can present an antitumor activity and a manageable safety as first-line treatment, and the study conducted by Vaishampayan et al. also showed 
greater results in the use of avelumab as first-line treatment $(57,58)$. Subsequently, Javelin Renal 101 confirmed the efficacy and safety of the combination of avelumab plus axitinib, when compared with sunitinib monotherapy, in terms of PFS while the data were still immature for OS - which is the main reason why this combination is not contemplated on the last guidelines $(59,60)$.

IMmotion 151 demonstrated a favourable safety profile with the combination (avelumab plus axitinib) over sunitinib, but once again the data were immature to conclude a benefit in OS $(61,62)$.

Some studies on the use of immune checkpoint inhibitors (ICI) have shown better results in populations with the PD-L1+, however, this does not make the expression of PD-L1 an effective biomarker for predicting the response to anti PD-1/ PD-L1 pathway. Therefore, studies demonstrated that the expression of PD-L1 may be associated with both poor prognosis and better responses to therapy. One of the theories attempt to explain this condition, defends that PD-L1 is a dynamic marker that can be regulated by cytokines induced by local inflammation, thus the expression of PDL1 within the tumour can change over time and according to the microenvironment conditions (59). Other biomarkers, such as the level of total cholesterol (TC) and the expression of sodium-dependent glucose transporter 2 (SGLT-2), have been studied in non-immunological therapies (63, 64). Future studies might focus on the validation of these biomarkers in immunotherapy.

The AE profile is also a condition with great impact on choosing a treatment to be used and it has also impact in the quality of patients' lives. The results show that the AE profile is similar between drugs from the same family, and in combinations, AE of both classes were observed. Although studies have shown a lower percentage pertaining to the occurrence of AE with ICI when compared to targeted therapy and conventional chemotherapy, ICI has a toxicity spectrum often associated with the immune system (irAE) $(38,46)$. Several studies showed a relation between these therapies and the occurrence of auto-immune events $(54,55,57,65)$. IrAE may include endocrine, dermatologic, gastrointestinal, hepatic, and other inflammatory events. Regarding PD-1/PDL1 inhibitors, dermatologic toxicity is the most reported and diarrhoea and colitis may be the most clinically relevant irAE in CTLA-4 inhibitors therapy, which have also led to death (38).

Vaishampayan et al. reported that the most commonly irAE were thyroid disorders (16.1\%) and immune-related rash (14.4\%) (57). De Giorgi et al. conclude, in a study focused on analysing the safety and efficacy of nivolumab, that in all the $\mathrm{AE}$ cases, 50\% were considered irAE (diarrhoea, hyperglycaemia, pneumonitis, asthenia, hypertension, skin toxicity, tremor, eyelid ptosis, liver toxicity and hypothyroidism) (65). Studies with pembrolizumab presented colitis, thyroiditis, hypothyroidism, adrenal insufficiency and hyperthyroidism as the most reported $\operatorname{irAE}(54,55)$.

Interestingly, in some cases, the occurrence of $\mathrm{AE}$ was associated to better outcomes. Although the reasons for this association are not clearly known, some hypotheses were postulated. It is believed that ICI can cause an immune system unbalance by their cross-reactivity with neoantigens and normal tissue antigens. Another theory defends that increased efficacy in patients with $\mathrm{AE}$ may be associated to the interaction between immunotherapy and polymorphisms in genes associated with ICI response. Since PD-1/PD-L1 inhibitors are implicated in the regulation of humoral immunity and influence the production of B cells, altered antibody production may also develop $\mathrm{AE}$ (46).

\section{CONCLUSIONS}

The recent years have been critical for the treatment of aRCC. A recent class of drugs, the ICI, showed advantages, with a greater OS, also providing an acceptable quality of life. This class of drugs is already the preconized first-line therapy, in combination with the previously used tyrosine kinase inhibitors or combining two different ICI drugs. The benefits of having a combined therapy are consequent dose reduction and, the reduction of irAE, with the capability to act in different pathways, increasing the treatment efficacy. While some combination regimens wait for mature results, the use of the current first-line therapies as 
the comparator in the trials will be mandatory and will certainly help us discover new therapeutic options for aRCC cancer patients.

Certainly, immunotherapy has greatly improved treatment of patients with aRCC, however, future studies should, in addition to effectiveness, also focus on ways to reduce toxicity.

\section{ABBREVIATIONS}

$\mathrm{AE}=$ adverse events

APC $=$ antigen-presenting cells

aRCC $=$ advanced renal cell carcinoma

ATE $=$ atezolizumab

AVE $=$ avelumab

AXI = axitinib

$\mathrm{BEV}=$ bevacizumab

CcRCC $=$ clear cell renal cell carcinoma

$\mathrm{CTL}=$ cytotoxic $\mathrm{T}$ lymphocytes

CTLA-4 = cytotoxic T lymphocyte associated protein 4

$\mathrm{DC}=$ dendritic cells

$\mathrm{EVE}=$ everolimus

$\mathrm{FAB}=$ variable region

$\mathrm{FC}=$ constant region

FDA $=$ Food and Drug Administration

GM-CSF = granulocyte-macrophage colony-stimu-

lating factor

HD IL-2 = high doses of IL-2

HLA = human lymphocyte antigens

ICI = immune checkpoint inhibitors

IFN- $\alpha=$ interferon alpha

IL-2 = interleukin-2

IMCD = International Metastatic RCC Database

Consortium

IPI = ipilimumab

ISTM = immunoreceptor tyrosine-bases switch motif

ITIM = immunoreceptor tyrosine-bases inhibitory motif

ITT = intention to treat

$\mathrm{Mab}=$ monoclonal antibodies

Mesh $=$ medical subject headings

MHC = major histocompatibility complex

$\mathrm{NIV}=$ nivolumab

$\mathrm{NK}=$ natural killer

$\mathrm{ORR}=$ objective rate response

OS $=$ overall survival

$\mathrm{PAZ}=$ pazopanib

PD-1 = programmed cell death protein

PD-L1 = programmed death-ligand 1
PEM = pembrolizumab

PFS $=$ progression-free survival

PPE $=$ palmar-plantar erythrodysthesia

$\mathrm{RCC}=$ renal cell carcinoma

SUN $=$ sunitinib

$\mathrm{TCR}=\mathrm{T}$ cell receptor

$\mathrm{TLR}=$ toll like receptors

VHL $=$ Von Hippel-Lindau

FUNDING INFORMATION

Ministério da Educação e Ciência, Fundação

para a Ciência e a Tecnologia

UID/NEU/04539/2013; UID/NEU/04539/2019);

UIDB/04539/2020; UIDP/04539/2020

\section{CONFLICT OF INTEREST}

None declared.

\section{REFERENCES}

1. Bray F, Ferlay J, Soerjomataram I, Siegel RL, Torre LA, Jemal A. Global cancer statistics 2018: GLOBOCAN estimates of incidence and mortality worldwide for 36 cancers in 185 countries. CA Cancer J Clin. 2018; 68:394-424. Erratum in: CA Cancer J Clin. 2020; 70:313.

2. Petejova N, Martinek A. Renal cell carcinoma: Review of etiology, pathophysiology and risk factors. Biomed Pap Med Fac Univ Palacky Olomouc Czech Repub. 2016; 160:183-94.

3. Ljungberg B, Bensalah K, Canfield S, Dabestani S, Hofmann $\mathrm{F}$, Hora $\mathrm{M}$, et al. EAU guidelines on renal cell carcinoma: 2014 update. Eur Urol. 2015; 67:913-24.

4. Suba I Ö, Aziret M, Karaman K, Ercan M. Colonic metastasis of renal cell carcinoma following curative nephrectomy: A case report and review of the literature. Int J Surg Case Rep. 2019;65:152-5

5. Capitanio U, Bensalah K, Bex A, Boorjian SA, Bray F, Coleman $\mathrm{J}$, et al. Epidemiology of Renal Cell Carcinoma. Eur Urol. 2019; 75:74-84.

6. Al-Marrawi MY, Holder SL. Current Immunotherapies for Renal Cell Carcinoma. Curr Mol Pharmacol. 2016;9:252-63.

7. Kruger S, IImer M, Kobold S, Cadilha BL, Endres S, Ormanns S, et al. Advances in cancer immunotherapy 2019 - latest trends. J Exp Clin Cancer Res. 2019; 38:268.

8. Zhang L, Huang Y, Lindstrom AR, Lin TY, Lam KS, Li Y. Peptide-based materials for cancer immunotherapy. Theranostics. 2019; 9:7807-25.

9. Pierce GN: Cancer immunotherapy - a promising approach Immunothérapie. Can J Physiol Pharmacol. 2018; 96:3-4. 
10. Trapani JA, Darcy PK. Immunotherapy of cancer. Aust Fam Physician. 2017; 46:194-199.

11. Riley RS, June $\mathrm{CH}$, Langer R, Mitchell MJ. Delivery technologies for cancer immunotherapy. Nat Rev Drug Discov. 2019; 18:175-96.

12. Zhang L, Tai YT, Ho MZG, Qiu L, Anderson KC. Interferonalpha-based immunotherapies in the treatment of $B$ cellderived hematologic neoplasms in today's treat-to-target era. Exp Hematol Oncol. 2017; 6:20.

13. Gill D, Hahn AW, Sonpavde G, Agarwal N. Immunotherapy of advanced renal cell carcinoma: Current and future therapies. Hum Vaccin Immunother. 2016; 12:2997-3004.

14. Mortara L, Balza E, Bruno A, Poggi A, Orecchia P, Carnemolla B. Anti-cancer Therapies Employing IL-2 Cytokine Tumor Targeting: Contribution of Innate, Adaptive and Immunosuppressive Cells in the Anti-tumor Efficacy. Front Immunol. 2018;9:2905.

15. Boyman 0, Arenas-Ramirez N. Development of a novel class of interleukin-2 immunotherapies for metastatic cancer. Swiss Med Wkly. 2019;149:w14697.

16. Sönmez MG, Sönmez LÖ. New treatment modalities with vaccine therapy in renal cell carcinoma. Urol Ann. 2019; 11:119-25.

17. Grenier JM, Yeung ST, Khanna KM. Combination Immunotherapy: Taking Cancer Vaccines to the Next Level. Front Immunol. 2018; 9:610.

18. Zhang R, Billingsley MM, Mitchell MJ. Biomaterials for vaccine-based cancer immunotherapy. J Control Release. 2018; 292:256-76.

19. Kartikasari AER, Prakash MD, Cox M, Wilson K, Boer JC, Cauchi JA, et al. Therapeutic Cancer Vaccines-T Cell Responses and Epigenetic Modulation. Front Immunol. 2019; 9:3109.

20. Kumai T, Fan A, Harabuchi Y, Celis E. Cancer immunotherapy: moving forward with peptide T cell vaccines. Curr Opin Immunol. 2017; 47:57-63.

21. Cho YH, Kim MS, Chung HS, Hwang EC. Novel immunotherapy in metastatic renal cell carcinoma. Investig Clin Urol. 2017; 58:220-7.

22. Amin A, Dudek AZ, Logan TF, Lance RS, Holzbeierlein JM, Knox JJ, et al. Survival with AGS-003, an autologous dendritic cell-based immunotherapy, in combination with sunitinib in unfavorable risk patients with advanced renal cell carcinoma (RCC): Phase 2 study results. J Immunother Cancer. $2015 ; 3: 14$.

23. Pan RY, Chung WH, Chu MT, Chen SJ, Chen HC, Zheng L, et al. Recent Development and Clinical Application of Cancer Vaccine: Targeting Neoantigens. J Immunol Res. 2018; 2018:4325874.
24. Rini BI, Stenzl A, Zdrojowy R, Kogan M, Shkolnik M, Oudard $S$, et al. IMA901, a multipeptide cancer vaccine, plus sunitinib versus sunitinib alone, as first-line therapy for advanced or metastatic renal cell carcinoma (IMPRINT): a multicentre, open-label, randomised, controlled, phase 3 trial. Lancet Oncol. 2016;17:1599-611.

25. Barata PC, Rini BI. Treatment of renal cell carcinoma: Current status and future directions. CA Cancer J Clin. 2017; 67:50724.

26. Pento JT. Monoclonal Antibodies for the Treatment of Cancer. Anticancer Res. 2017; 37:5935-9.

27. Bayer V. An Overview of Monoclonal Antibodies. Semin Oncol Nurs. 2019; 35:150927.

28. Koo SL, Wang WW, Toh HC. Cancer Immunotherapy - The Target is Precisely on The Cancer and Also Not. Ann Acad Med Singapore. 2018; 47:381-7.

29. Witkowska M, Smolewski P. Immune Checkpoint Inhibitors to Treat Malignant Lymphomas. J Immunol Res. 2018; 2018:1982423.

30. Atkins MB, Clark JI, Quinn DI. Immune checkpoint inhibitors in advanced renal cell carcinoma: experience to date and future directions. Ann Oncol. 2017; 28:1484-94.

31. D'Angelo SP, Kelly CM: Immune Checkpoint Inhibitors in Sarcoma. Immunother. Sarcoma, Springer Int Publishing. 2019; 42: pp. 125-37.

32. Hargadon KM, Johnson CE, Williams CJ. Immune checkpoint blockade therapy for cancer: An overview of FDA-approved immune checkpoint inhibitors. Int Immunopharmacol. 2018; 62:29-39.

33. McNeel DG. Therapeutic Cancer Vaccines: How Much Closer Are We? BioDrugs. 2018; 32:1-7.

34. Bashir B, Wilson MA. Novel Immunotherapy Combinations. Curr Oncol Rep. 2019; 21:96.

35. Akce M, Zaidi MY, Waller EK, El-Rayes BF, Lesinski GB. The Potential of CAR T Cell Therapy in Pancreatic Cancer. Front Immunol. 2018; 9:2166.

36. Rowshanravan B, Halliday N, Sansom DM. CTLA-4: a moving target in immunotherapy. Blood. 2018; 131:58-67.

37. Rizzo M, Alaniz L, Mazzolini GD: Células presentadoras de antígeno profesionales: células dendríticas Uno de los importantes nexos entre la respuesta inmuni-. 2016:307-14.

38. Salmaninejad A, Valilou SF, Shabgah AG, Aslani $S$, Alimardani M, Pasdar A, et al. PD-1/PD-L1 pathway: Basic biology and role in cancer immunotherapy. J Cell Physiol. 2019; 234:16824-37.

39. Chen VJ, Hernandez-Meza G, Agrawal P, Zhang CA, Xie L, Gong CL, et al. Time on Therapy for at Least Three Months Correlates with Overall Survival in Metastatic Renal Cell Carcinoma. Cancers (Basel). 2019; 11:1000. 
40. Arranz-Nicolás J, Mérida I. Biological regulation of diacylglycerol kinases in normal and neoplastic tissues: New opportunities for cancer immunotherapy. Adv Biol Regul. 2020; 75:100663.

41. Wei C, Wang S, Ye Z, Chen Z. Efficacy of targeted therapy for advanced renal cell carcinoma: a systematic review and meta-analysis of randomized controlled trials. Int Braz $\mathrm{J}$ Urol. 2018; 44:219-37.

42. Curti B, Daniels GA, McDermott DF, Clark JI, Kaufman HL, Logan TF, et al. Improved survival and tumor control with Interleukin-2 is associated with the development of immunerelated adverse events: data from the PROCLAIMSM registry. $\mathrm{J}$ Immunother Cancer. 2017; 5:102.

43. Eto M, Kawano Y, Hirao Y, Mita K, Arai Y, Tsukamoto T, et al. Phase II clinical trial of sorafenib plus interferon-alpha treatment for patients with metastatic renal cell carcinoma in Japan. BMC Cancer. 2015; 15:667.

44. Donskov F, Jensen NV, Smidt-Hansen T, Brøndum L, Geertsen P. A randomized phase II trial of interleukin-2 and interferon- $\alpha$ plus bevacizumab versus interleukin-2 and interferon- $\alpha$ in metastatic renal-cell carcinoma (mRCC): results from the Danish Renal Cancer Group (DaRenCa) study-1. Acta Oncol. 2018;57:589-94.

45. Motzer RJ, Escudier B, McDermott DF, George S, Hammers HJ, Srinivas S, et al. Nivolumab versus Everolimus in Advanced Renal-Cell Carcinoma. N Engl J Med. 2015; 373:1803-13.

46. Verzoni E, Cartenì G, Cortesi E, Giannarelli D, De Giglio A, Sabbatini $R$, et al. Real-world efficacy and safety of nivolumab in previously-treated metastatic renal cell carcinoma, and association between immune-related adverse events and survival: the Italian expanded access program. J Immunother Cancer. 2019; 7:99.

47. Stukalin I, Wells JC, Graham J, Yuasa T, Beuselinck B, Kollmansberger $\mathrm{C}$, et al. Real-world outcomes of nivolumab and cabozantinib in metastatic renal cell carcinoma: results from the International Metastatic Renal Cell Carcinoma Database Consortium. Curr Oncol. 2019; 26:e175-e179.

48. Kimura M, Usami E, Teramachi H, Yoshimura T. A comparative study of nivolumab and axitinib in terms of overall survival treatment continuation, and cost for patients with metastatic renal cell carcinoma. Mol Clin Oncol. 2020; 12:284-9.

49. Amin A, Plimack ER, Ernstoff MS, Lewis LD, Bauer TM, McDermott DF, et al. Safety and efficacy of nivolumab in combination with sunitinib or pazopanib in advanced or metastatic renal cell carcinoma: the CheckMate 016 study. J Immunother Cancer. 2018; 6:109. Erratum in: J Immunother Cancer. 2019; 7:73.

50. Hammers HJ, Plimack ER, Infante JR, Rini BI, McDermott DF, Lewis LD, et al. Safety and Efficacy of Nivolumab in Combination With Ipilimumab in Metastatic Renal Cell Carcinoma: The CheckMate 016 Study. J Clin Oncol. 2017; 35:3851-8.
51. Tomita $Y$, Kondo T, Kimura G, Inoue T, Wakumoto $Y$, Yao M, et al. Nivolumab plus ipilimumab versus sunitinib in previously untreated advanced renal-cell carcinoma: analysis of Japanese patients in CheckMate 214 with extended follow-up. Jpn J Clin Oncol. 2020; 50:12-9.

52. Rini BI, Battle D, Figlin RA, George DJ, Hammers H, Hutson $T$, et al. The society for immunotherapy of cancer consensus statement on immunotherapy for the treatment of advanced renal cell carcinoma (RCC). J Immunother Cancer. 2019; 7:354.

53. Rini BI, Plimack ER, Stus V, Gafanov R, Hawkins R, Nosov $D$, et al. Pembrolizumab plus Axitinib versus Sunitinib for Advanced Renal-Cell Carcinoma. N Engl J Med. 2019; 380:1116-27.

54. Atkins MB, Plimack ER, Puzanov I, Fishman MN, McDermott DF, Cho DC, et al. Axitinib in combination with pembrolizumab in patients with advanced renal cell cancer: a non-randomised, open-label, dose-finding, and doseexpansion phase 1b trial. Lancet Oncol. 2018; 19:405-15.

55. Taylor MH, Lee CH, Makker V, Rasco D, Dutcus CE, Wu J, et al. Phase IB/II Trial of Lenvatinib Plus Pembrolizumab in Patients With Advanced Renal Cell Carcinoma, Endometrial Cancer, and Other Selected Advanced Solid Tumors. J Clin Oncol. 2020; 38:1154-63. Erratum in: J Clin Oncol. 2020; 38:2702.

56. Wang B, Gu W, Wan F, Shi G, Ye D. Prognostic significance of the dynamic changes of systemic inflammatory response in metastatic renal cell carcinoma. Int Braz J Urol. 2019; 45:89-99.

57. Vaishampayan U, Schöffski P, Ravaud A, Borel C, Peguero $\mathrm{J}$, Chaves $\mathrm{J}$, et al. Avelumab monotherapy as first-line or second-line treatment in patients with metastatic renal cell carcinoma: phase Ib results from the JAVELIN Solid Tumor trial. J Immunother Cancer. 2019; 7:275.

58. Choueiri TK, Larkin J, Oya M, Thistlethwaite F, Martignoni M, Nathan $\mathrm{P}$, et al. Preliminary results for avelumab plus axitinib as first-line therapy in patients with advanced clear-cell renal-cell carcinoma (JAVELIN Renal 100): an open-label, dose-finding and dose-expansion, phase $1 \mathrm{~b}$ trial. Lancet Oncol. 2018; 19:451-60.

59. Primo N. Lara EJ: Kidney cancer. Second Eds. Springer. 2017; 16: pp. 268-79.

60. Motzer RJ, Penkov K, Haanen J, Rini B, Albiges L, Campbell MT, et al. Avelumab plus Axitinib versus Sunitinib for Advanced Renal-Cell Carcinoma. N Engl J Med. 2019; 380:1103-15.

61. Rini BI, Powles T, Atkins MB, Escudier B, McDermott DF Suarez $\mathrm{C}$, et al. Atezolizumab plus bevacizumab versus sunitinib in patients with previously untreated metastatic renal cell carcinoma (IMmotion151): a multicentre, openlabel, phase 3, randomised controlled trial. Lancet. 2019; 393:2404-15. 
62. McDermott DF, Huseni MA, Atkins MB, Motzer RJ, Rini BI, Escudier B, et al. Clinical activity and molecular correlates of response to atezolizumab alone or in combination with bevacizumab versus sunitinib in renal cell carcinoma. Nat Med. 2018; 24:749-57. Erratum in: Nat Med. 2018; 24:1941.

63. Kobayashi M, Uematsu T, Tokura Y, Takei K, Sakamoto K, Narimatsu $\mathrm{T}$, et al. Immunohistochemical expressionof sodium-dependent glucose transporter - 2 (SGLT-2) in clear cell renal carcinoma: possible prognostic implications. Int Braz J Urol. 2019; 45:169-78.

\section{Fernando Mendes, MD}

Escola Superior de Tecnologia da Saúde de Coimbra Rua 5 de Outubro / 7006, SM Bispo

Coimbra, 3046-854, Portugal

E-mail:fjmendes@estescoimbra.pt
64. Li B, Huang D, Zheng H, Cai Q, Guo Z, Wang S. Preoperative serum total cholesterol is a predictor of prognosis in patients with renal cell carcinoma: a meta- analysis of observational studies. Int Braz J Urol. 2020; 46:158-68.

65. De Giorgi U, Cartenì G, Giannarelli D, Basso U, Galli L, Cortesi $E$, et al. Safety and efficacy of nivolumab for metastatic renal cell carcinoma: real-world results from an expanded access programme. BJU Int. 2019; 123:98-105.

Submitted for publication:

July 28,2020

Accepted after revision: August 05, 2020

Published as Ahead of Print:

January 20, 2021 